\title{
GENERALIZED COPRIME ARRAY CONFIGURATIONS
}

\author{
Si Qin, Yimin D. Zhang, and Moeness G. Amin \\ Center for Advanced Communications, Villanova University, Villanova, PA 19085, USA
}

\begin{abstract}
A coprime array consists of two uniform linear subarrays that construct an effective difference co-array with certain desirable characteristics. In this paper, we propose a generalized coprime array concept through the compression of the interelement spacing of one constituting subarray. As such, the existing variations of coprime array and nested array structures are represented as special cases. The achievable unique lags as well as consecutive lags in the resulting virtual array are analytically expressed, and the direction-of-arrival estimation performance is examined using both the MUSIC algorithm and sparse signal reconstruction techniques.
\end{abstract}

Index Terms - Coprime array, sparse array, difference co-array, direction-of-arrival estimation, compressive sensing

\section{INTRODUCTION}

Direction-of-arrival (DOA) estimation, which determines the spatial spectra of the impinging electromagnetic waves, is an important application area of antenna arrays. It is known that conventional DOA estimation methods, such as MUSIC and ESPRIT [1, 2], resolve up to $N-1$ sources with an $N$-element array. The problem of detecting more sources than the number of sensors, on the other hand, is of tremendous interest in various applications [3]. Virtual arrays with a higher number of degrees-of-freedom (DOFs) can be achieved from sparse array structures under the difference co-array equivalence. The minimum redundancy array (MRA) [4] is a wellknown early configuration of linear array which, for a given number of physical sensors, maximizes the number of consecutive virtual sensors in the resulting difference co-array. There is no systematical method to obtain the MRA configurations for an arbitrary number of sensors and to assess the corresponding achievable DOFs. Therefore, the optimum design and performance analysis of such arrays is not straightforward.

Recently, several array configurations have been proposed as attractive alternatives for sparse array construction. The nested array [6] can resolve $O\left(N^{2}\right)$ sources with only $O(N)$ sensors, and is obtained by combining two uniform linear sub-arrays where one subarray assumes a unit interelement spacing. Unlike MRAs, the nested array configuration is easy to construct and it is possible to provide exact expressions for the sensor locations and the available DOFs for a given number of the sensors. The total aperture and the number of unique and consecutive co-array sensors can be subsequently obtained [6]. The coprime array developed in [7] which utilizes a coprime pair of uniform linear subarrays is another attractive sparse array structure. A standard coprime array, which is referred to as the

This work was supported in part by the Office of Naval Research (ONR) under grant N00014-13-1-0061. prototype coprime array in this paper, consists of two uniform subarrays, where one is of $M$ sensors with an interelement spacing of $N$ units, and the other is of $N$ elements with an interelement spacing of $M$ units. Note that $M$ and $N$ are required to be coprime positive integers. A coprime array with $M+N-1$ sensors can resolve up to $O(M N)$ sources.

The main objectives of this paper is to generalize the coprime and nested array configurations developed in $[6,7,8]$, and derive the precise expressions of the achievable number of virtual array sensors. In particular, we introduce the concept of generalized coprime array (GCA), which permits an integer compression factor to be applied to the interelement spacing of one constituting subarray. As such, the two coprime array structures developed in [7,8] as well as the nested array structure developed in [6] become special cases of the proposed generalized coprime arrays. The performance of the GCA structures is evaluated using their difference co-array equivalence. The analytical expressions of the co-array aperture and the numbers of unique lags as well as consecutive lags are given for quantitative evaluation and optimal design.

Notations: We use lower-case (upper-case) bold characters to denote vectors (matrices). In particular, $\mathbf{I}_{N}$ denotes the $N \times N$ identity matrix. (.)* implies complex conjugation, whereas $(.)^{T}$ and $(.)^{H}$ respectively denote the transpose and conjugate transpose of a matrix or vector. In addition, $\operatorname{vec}(\cdot)$ denotes the vectorization operator that turns a matrix into a vector by stacking all columns on top of the another, and $\operatorname{diag}(\mathbf{x})$ denotes a diagonal matrix that uses the elements of $\mathbf{x}$ as its diagonal elements. $\|\cdot\|_{2}$ and $\|\cdot\|_{1}$ respectively denote the Euclidean $\left(l_{2}\right)$ and $l_{1}$ norms, and $\mathrm{E}(\cdot)$ is the statistical expectation operator. $\otimes$ denotes the Kronecker product, and real $(\cdot)$ and $\operatorname{imag}(\cdot)$ respectively represent the real and imaginary part operations.

\section{COPRIME ARRAY CONCEPT}

A prototype coprime array, as described in the previous section, is illustrated in Fig. 1. Without loss of generality, we assume $M<N$. The unit interelement spacing is set to $d=\lambda / 2$. The array sensors are positioned at

$$
P=\{M n d, 0 \leq n \leq N-1\} \cup\{N m d, 0 \leq m \leq M-1\} .
$$

Because the two subarrays share the first sensor at the zeroth position, the total number of the sensors used in the coprime array is $M+N-1$. Note that the minimum interelement spacing in this coprime array is $d$.

Denote $\mathbf{p}=\left[p_{1}, \ldots, p_{M+N-1}\right]^{T}$ as the positions of the array sensors where $p_{i} \in P, i=1, \ldots, M+N-1$. The first sensor is assumed as the reference, i.e., $p_{1}=0$. Assume that $Q$ uncorrelated signals imping on the array from angles $\Theta=\left[\theta_{1}, \ldots, \theta_{Q}\right]^{T}$, and their discritized baseband waveforms are expressed as $s_{q}(t), t=1, \ldots, T$, 


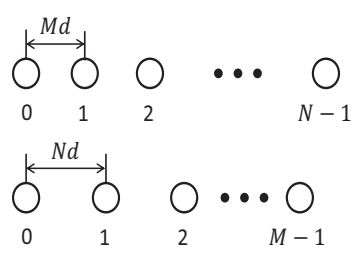

Fig. 1. The prototype coprime array configuration.

for $q=1, \ldots, Q$. Then, the data vector received at the coprime array is expressed as

$$
\mathbf{x}(t)=\sum_{q=1}^{Q} \mathbf{a}\left(\theta_{q}\right) s_{q}(t)+\mathbf{n}(t)=\mathbf{A} \mathbf{s}(t)+\mathbf{n}(t),
$$

where

$$
\mathbf{a}\left(\theta_{q}\right)=\left[1, e^{j \frac{2 \pi p_{2}}{\lambda} \sin \left(\theta_{q}\right)}, \ldots, e^{j \frac{2 \pi p_{M+N}-1}{\lambda} \sin \left(\theta_{q}\right)}\right]^{T}
$$

is the steering vector of the array corresponding to $\theta_{q}, \mathbf{A}=$ $\left[\mathbf{a}\left(\theta_{1}\right), \ldots, \mathbf{a}\left(\theta_{Q}\right)\right]$, and $\mathbf{s}(t)=\left[s_{1}(t), \ldots, s_{Q}(t)\right]^{T}$. The elements of the noise vector $\mathbf{n}(t)$ are assumed to be independent and identically distributed (i.i.d.) random variables following the complex Gaussian distribution $\mathcal{N} C\left(0, \sigma_{n}^{2}\right)$.

The covariance matrix of data vector $\mathbf{x}(t)$ is obtained as

$$
\begin{aligned}
\mathbf{R}_{\mathbf{x x}} & =\mathrm{E}\left[\mathbf{x}(t) \mathbf{x}^{H}(t)\right]=\mathbf{A} \mathbf{R}_{\mathbf{s s}} \mathbf{A}^{H}+\sigma_{n}^{2} \mathbf{I}_{M+N-1} \\
& =\sum_{q=1}^{Q} \sigma_{q}^{2} \mathbf{a}\left(\theta_{q}\right) \mathbf{a}^{H}\left(\theta_{q}\right)+\sigma_{n}^{2} \mathbf{I}_{M+N-1},
\end{aligned}
$$

where $\mathbf{R}_{\mathbf{s s}}=\mathrm{E}\left[\mathbf{s}(t) \mathbf{s}^{H}(t)\right]=\operatorname{diag}\left(\left[\sigma_{1}^{2}, \ldots, \sigma_{Q}^{2}\right]\right)$ is the source covariance matrix, with $\sigma_{q}^{2}$ denoting the input signal power of the $q$ th source, $q=1, \ldots, Q$. In practice, the covariance matrix is estimated using the $T$ available samples.

From antennas located at the $i$ th and $k$ th positions in $\mathbf{p}$, the correlation $\mathrm{E}\left[x_{i}(t) x_{k}^{*}(t)\right]$ yields an entry in $\mathbf{R}_{\mathbf{x x}}$ with lag $p_{i}-p_{k}$. As such, all the available values of $i$ and $k$, where $0 \leq i \leq M+N-1$ and $0 \leq k \leq M+N-1$, yields virtual sensors of the following difference co-array:

$$
C_{P}=\{\mathbf{z} \mid \mathbf{z}=\mathbf{u}-\mathbf{v}, \mathbf{u} \in P, \mathbf{v} \in P\} .
$$

The significance of the difference co-array is that the correlation of the received signal can be calculated at all difference lags contained in set $C_{P}$. Such different lags form a virtual array at these lag positions to achieve increased number of degrees-of-freedom (DOFs) for DOA estimation. The number of unique elements in set $C_{P}$ directly determines the number of distinct cross-correlation lags in the covariance matrix, which is related to the number of DOFs. By using part or the entire set of the obtained virtual sensors, instead of the original array, to perform DOA estimation, we can increase the number of sources that can be detected by the array.

\section{DOA ESTIMATION}

To understand the role of unique and consecutive co-array lags in DOA estimation, we briefly summarize the MUSIC algorithm and compressive sensing (CS)-based approaches exploiting coprime arrays (refer to [8] and [9] for details).

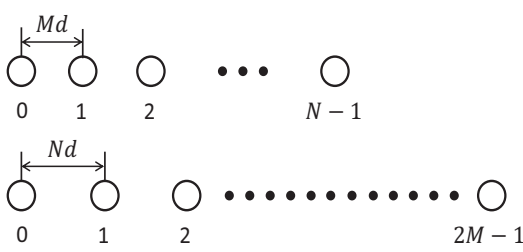

Fig. 2. The conventional coprime array configuration.

\subsection{MUSIC Algorithm}

Vectorizing $\mathbf{R}_{\mathbf{x x}}$ yields

$$
\mathbf{z}=\operatorname{vec}\left(\mathbf{R}_{\mathbf{x x}}\right)=\tilde{\mathbf{A}} \mathbf{b}+\sigma_{n}^{2} \tilde{\mathbf{i}}=\mathbf{B r}
$$

where $\tilde{\mathbf{A}}=\left[\tilde{\tilde{\mathbf{a}}}\left(\theta_{1}\right), \ldots, \tilde{\mathbf{a}}\left(\theta_{Q}\right)\right], \tilde{\mathbf{a}}\left(\theta_{q}\right)=\mathbf{a}^{*}\left(\theta_{q}\right) \otimes \mathbf{a}\left(\theta_{q}\right), \mathbf{b}=$ $\left[\sigma_{1}^{2}, \ldots, \sigma_{Q}^{2}\right]^{T}, \tilde{\mathbf{i}}=\operatorname{vec}\left(\mathbf{I}_{\tilde{M}+\tilde{N}-1}\right)$. In addition, $\mathbf{B}=[\tilde{\mathbf{A}}, \tilde{\mathbf{i}}]$ and $\mathbf{r}=\left[\mathbf{b}^{T}, \sigma_{n}^{2}\right]^{T}=\left[\sigma_{1}^{2}, \ldots, \sigma_{Q}^{2}, \sigma_{n}^{2}\right]$ are used to simplify the notations. The vector $\mathbf{z}$ amounts to the received data from a virtual array with an extended co-array aperture whose corresponding steering matrix is defined by $\tilde{\mathbf{A}}$. However, the virtual source signal becomes a single snapshot of $\mathbf{b}$. Therefore, the rank of the noise-free covariance matrix of $\mathbf{z}, \mathbf{R}_{\mathbf{z z}}=\mathbf{z z}^{H}$, is one, and subspace-based DOA estimation techniques, such as MUSIC, fail to yield DOA estimates when multiple signals impinge to the array.

To overcome the above problem, as shown in Fig. 2, it is proposed in [8] to double the number of array sensor in the first subarray, which has fewer sensors than the other. In this paper, we refer to this array structure as the conventional coprime array. In such a conventional coprime array, the number of sensors in the two subarrays are $N$ and $2 M$, respectively, where $M<N$. Because the zeroth sensor positions of the two arrays are collocated, the total number of the physical sensors in this coprime array structure is $N+2 M-1$. Note that the minimum interelement spacing remains $d$. This coprime array generates all the consecutive $2 M N+1$ lag differences from $-M N$ to $M N$ [8]. By extracting these lags, we can construct a $(2 M N+1) \times(2 M N+1)$ covariance matrix, denoted as $\mathbf{R}_{\mathbf{z z}}^{\prime}$. Performing spatial smoothing yields an $(M N+1) \times(M N+1)$ fullrank covariance matrix. The MUSIC algorithm can then be applied to estimate in theory DOAs, up to $M N$ sources.

\subsection{Compressive Sensing Approach}

Alternatively, (6) can be solved using the CS approach $[9,10]$. The desired result of $\mathbf{b}$ is represented as the solution to the following constrained $l_{1}$-norm minimization problem

$$
\hat{\mathbf{r}}^{\circ}=\arg \min _{\mathbf{r}^{\circ}}\left\|\mathbf{r}^{\circ}\right\|_{1} \quad \text { s.t. } \quad\left\|\mathbf{z}-\mathbf{B}^{\circ} \mathbf{r}^{\circ}\right\|_{2}<\epsilon,
$$

where $\epsilon$ is a user-specific bound, $\mathbf{B}^{\circ}$ is a sensing matrix consisting of the searching steering vectors, and $\mathbf{r}^{\circ}$ is a sparse vector to be determined with entries defined in these search grids.

This type of problems has been the objective of intensive studies in the area of CS, and a number of effective numerical computation methods have been developed. Similar to $[9,10]$, we use the batch Lasso algorithm [11, 12] in this paper, however other methods may also be used.

\section{GENERALIZED COPRIME ARRAY CONFIGURATIONS}

Now we consider the generalization of coprime array structures with two subarrays with $M$ and $N$ sensors, where $M$ and $N$ are coprime. 
Unlike the prototype coprime array, an integer compression factor $p$ is introduced for changing the interelement spacing of one subarray. Let

$$
M=p \tilde{M}, \quad N=\tilde{N},
$$

where $p$ is an integer and $2 \leq p \leq M$. It is easy to confirm that $\tilde{M}$ and $\tilde{N}$ are also coprime since $M$ and $N$ do not have common factors other than unity. It is also clear that the coprime array variation configuration in [8] is a special case of the generalized coprime configuration by choosing $p=2$.

As shown in Fig. 3, in the generalized coprime array, the $M$ element subarray has an interelement space of $\tilde{N} d=N d$, whereas the $N$-element subarray has an interelement space of $\tilde{M} d=M d / p$. As such, the generalized coprime array permits the interelement spacing of one constituting subarray to be compressed by an integer factor of $p$. Note that all arrays under this generalization consist of the same $M+N-1$ physical antenna sensors regardless of the value of $p$.

To gain more insights about this array configuration, we separately consider the self-lags of the two subarrays and their cross-lags. Denote

$$
L_{\mathrm{s}}=\{\tilde{M} n, 0 \leq n \leq N-1\} \cup\{\tilde{N} m, 0 \leq m \leq M-1\},
$$

and

$$
L_{\mathrm{c}}=\{\tilde{N} m-\tilde{M} n, 0 \leq n \leq N-1,0 \leq m \leq M-1\} .
$$

respectively, as the self- and the cross-lags.

Since $\mathbf{R}_{\mathrm{xx}}$ is a Hermitian matrix, $\left\{-L_{\mathrm{s}}\right\}$ and $\left\{-L_{\mathrm{c}}\right\}$ are also valid lag sets. Consequently, the overall lag set of the entire virtual array is given by

$$
L_{\mathrm{P}}=\left\{L_{\mathrm{s}}\right\} \cup\left\{L_{\mathrm{c}}\right\} \cup\left\{-L_{\mathrm{s}}\right\} \cup\left\{-L_{\mathrm{c}}\right\} .
$$

To completely exploit the DOFs of the GCA configuration, we summarize the properties of $L_{\mathrm{s}}$ and $L_{\mathrm{c}}$ in Proposition 1.

Proposition 1: The following facts hold for GCA:

(a) There are $M N$ distinct integers in set $L_{\mathrm{c}}$.

(b) $L_{\mathrm{c}}$ contains all the contiguous integers in the range $-(N-$ $1) \leq l_{c} \leq M N-\tilde{M}(N-1)-1$.

(c) The negative values in set $L_{\mathrm{c}}$ form a subset of the flipped positive counterpart, i.e., $\left\{l_{c}, l_{c}<0\right\} \subseteq\left\{-l_{c}, l_{c}>0\right\}$.

(d) $L_{\mathrm{s}} \subseteq L_{\mathrm{c}}$.

(e) The "holes" are located at $-(a \tilde{M}+b \tilde{N})$ in the negative range of $L_{\mathrm{c}}$, where $a \geq 0$ and $b>0$ are integers.

The proof is provided in [13].

Based on Proposition 1, the entire lags set in virtual array defined in (11) consists of $\left\{l_{c}, l_{c} \geq 0\right\} \cup\left\{-l_{c}, l_{c} \geq 0\right\}$, thus resulting in Proposition 2.

Proposition 2: The GCA configuration yields a virtual array such that:

(a) It contains $2 M N-(\tilde{M}+1)(N-1)-1$ unique lags of virtual sensors.

(b) Among them there are $2 M N-2 \tilde{M}(N-1)-1$ consecutive integers within the range of $[-M N+\tilde{M}(N-1)+1, M N-$ $\tilde{M}(N-1)-1]$.

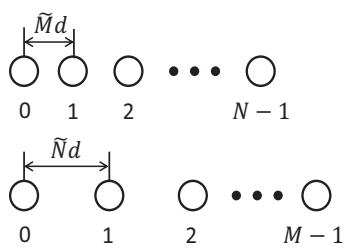

Fig. 3. The GCA configuration.

The proof is also provided in [13]. Note that our result contains more consecutive lags and is more precise than the result in [8] provided for the case of $M=2 \tilde{M}$.

According to Proposition 2, we can draw a conclusion that, for a specific pair of $M$ and $N$, the smaller $\tilde{M}$ is, the more unique and consecutive lags that virtual sensors have. In other words, both numbers increase with the compression factor $p$. The minimum value that $\tilde{M}$ can take is 1 . In this case, the GCA configuration becomes the nested array which provides the highest numbers of the unique and consecutive virtual sensors.

\section{SIMULATION RESULTS}

For illustrative purposes, we consider $M=6$ and $N=7$ with different values of the compression factor $p$, as shown in Fig. 4. All configurations consist of 12 physical antenna sensors and the unit interelement spacing is $d=\lambda / 2$. Fig. 4(a) depicts the example for $p=2$ where the coprime array forms a virtual array with 59 unique lags, among which 47 lags within $[-23,23]$ are consecutive. Note that there are holes in the difference co-array that are indicated by " $x$ " in the figure. Fig. 4(b) shows for the case of $p=3$, and the resulting virtual array has 65 unique lags, among which 59 lags with $[-29,29]$ are consecutive. When $p=M=6$, i.e., $\tilde{M}=1$, as shown in Fig. 4(c), the coprime array becomes the nested array structure with 71 unique lags, which are all consecutive. It is clear that both numbers of the unique and consecutive lags increase with $p$, and the nested array achieves the maximum number for both.

To compare the DOA estimation performance, we first consider a relatively clean covariance matrix by assuming 2000 noise-free snapshots in order to fully explore the DOFs offered by the resulting virtual arrays. $Q=33$ uncorrelated narrowband sources are considered, which are uniformly distributed between $-60^{\circ}$ and $60^{\circ}$. In Figs. 5(a) and 5(b), we respectively obtained 29 and 35 DOFs for $p=3$ and $p=6$ when applying the MUSIC algorithm, which only exploits consecutive virtual array lags. Note that only the latter has sufficient DOFs to resolve all 33 impinging signals. This is verified in the two plots as only the nested array $(p=6)$ resolves all the 33 signals, whereas not all sources are correctly identified for the case of $p=3$. Due to space limitation, the result for $p=2$ is not shown as it has even lower number of DOFs than the $p=3$ case. For CS, a higher number of DOFs is achieved because all unique lags are exploited. The results obtained from the Lasso are shown in Figs. 5(c) and 5(d), where a grid interval of $\theta_{i}^{\mathrm{g}}=0.25^{\circ}$ is used to perform. The results further demonstrate the optimality of the nested array. In addition, it is clearly shown that, while both MUSIC and CS based methods resolve all 33 signals for the nested array structure, the CSbased technique results in better estimated spectra.

Next, the performance of GCA configurations is considered in the presence of noise with a $0 \mathrm{~dB}$ SNR for all signals, and the number of snapshots is reduced to 500. In this case, the disturbance in the 
covariance matrix becomes higher due to noise and the limited number of samples. The DOA estimation results are compared in Fig. 6 for $Q=26$ sources, which is smaller than the available DOFs for both array configurations under $p=3$ and $p=6$. It is evident that the latter outperforms the former. As a comparison, the CS based method obtains better spatial spectra.

\section{CONCLUSIONS}

We proposed a general coprime array structure, termed GCA, for direction-of-arrival (DOA) estimations. The proposed configurations can exploit different compression factors that reduce the interelement spacing of one constituting subarray. The resulting difference co-array aperture, the number of unique lags, and the number of consecutive lags are analytically given. A quantitative evaluation is provided by demonstrating the capability of DOA estimation of a high number of sources, which is consistent with the increased number of virtual sensors.

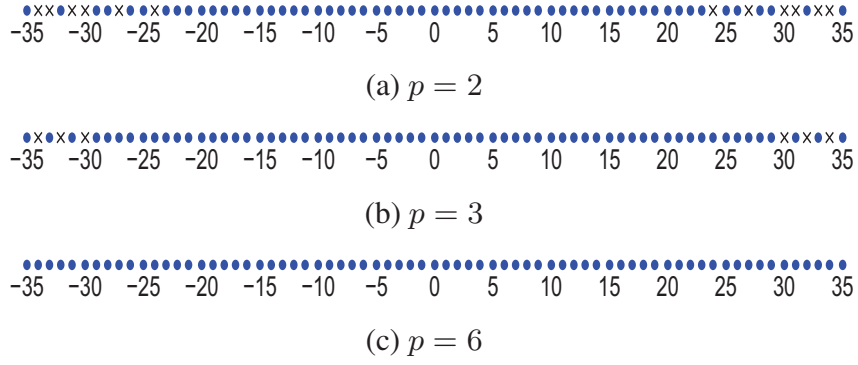

Fig. 4. Virtual sensor positions for GCA configurations with different values of compression factor $p(M=6$ and $N=7)$.

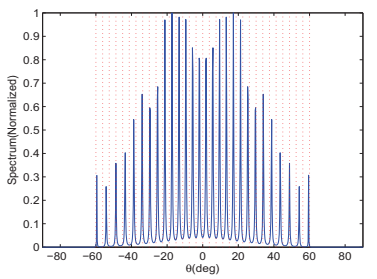

(a) MUSIC with $p=3$

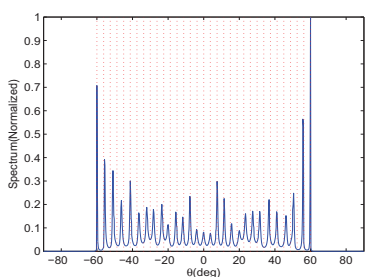

(c) Lasso with $p=3$

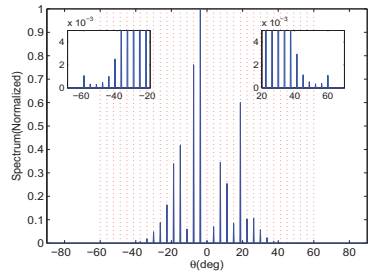

(b) MUSIC with $p=6$

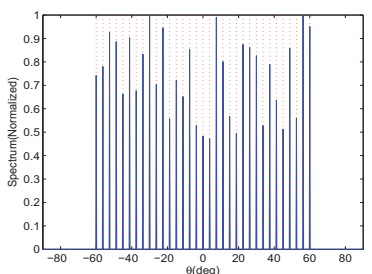

(d) Lasso with $p=6$
Fig. 5. Estimated spatial spectra (noise-free, 2000 snapshots, $Q=33$ ).

\section{REFERENCES}

[1] R. O. Schmidt, "Multiple emitter location and signal parameter estimation," IEEE Trans. Antennas Propagat., vol. 34, no. 3, pp. 276-280, March 1986.

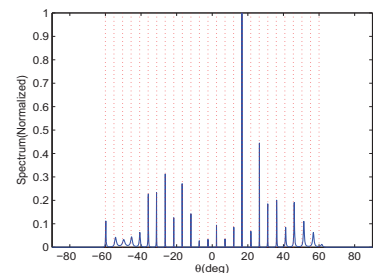

(a) MUSIC with $p=3$

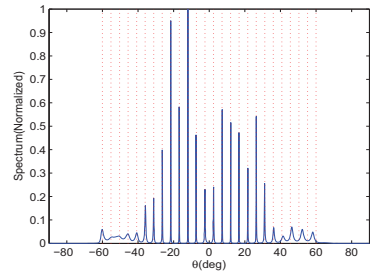

(a) Lasso with $p=3$

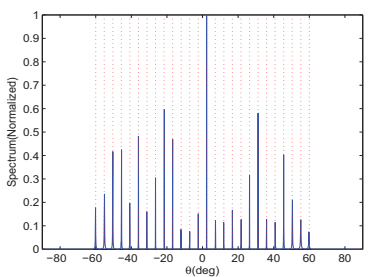

(b) MUSIC with $p=6$

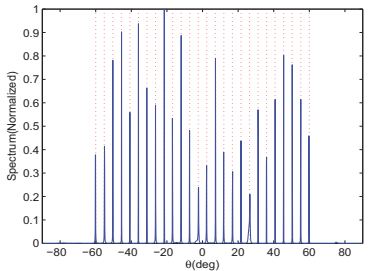

(b) Lasso with $p=6$
Fig. 6. Estimated spatial spectra ( $\mathrm{SNR}=0 \mathrm{~dB}, 500$ snapshots, $Q=26)$.

[2] R. Roy and T. Kailath, "ESPRIT - Estimation of signal parameters via rotation invariance techniques," IEEE Trans. Acoust., Speech, Signal Proc., vol. 17, no. 7, pp. 984-995, July 1989.

[3] R. T. Hoctor and S. A. Kassam, "The unifying role of the coarray in aperture synthesis for coherent and incoherent imaging," Proc. IEEE, vol. 78, no. 4, pp. 735-752, April 1990.

[4] A. Moffet, "Minimum-redundancy linear arrays," IEEE Trans. Antennas Propagat., vol. 16, no. 2, pp. 172-175, March 1968.

[5] G. S. Bloom and S. W. Golomb, "Application of numbered undirected graphs," Proc. IEEE, vol. 65, no. 4, pp. 562-570, April 1977.

[6] P. Pal and P. P. Vaidyanathan, "Nested Arrays: A novel approach to array processing with enhanced degrees of freedom," IEEE Trans. Signal Proc., vol. 58, no. 8, pp. 4167-4181, Aug. 2010.

[7] P. P. Vaidyanathan and P. Pal, "Sparse sensing with co-prime samplers and arrays," IEEE Trans. Signal Proc., vol. 59, no. 2, pp. 573-586, Feb. 2011.

[8] P. Pal and P. P. Vaidyanathan, "Coprime sampling and the MUSIC algorithm," in Proc. IEEE Digital Signal Proc. Workshop and IEEE Signal Proc. Education Workshop, Sedona, AZ, Jan. 2011.

[9] Y. D. Zhang, M. G. Amin, and B. Himed, "Sparsity-based DOA estimation using co-prime arrays," in Proc. IEEE ICASSP, Vancouver, Canada, May 2013.

[10] Y. D. Zhang, S. Qin, and M. G. Amin, "DOA estimation exploiting coprime arrays with sparse sensor spacing," in Proc. IEEE ICASSP, Frorence, Italy, May 2014.

[11] P. Pal and P. P. Vaidyanathan, "On application of LASSO for sparse support recovery with imperfect correlation awareness," in Proc. Asilomar Conf. Signals, Systems and Computers, Pacific Grove, CA, Nov. 2012.

[12] R. Tibshirani, "Regression shrinkage and selection via the lasso," J. Royal Statistical Society, Series B, vol. 58, no. 1, pp. 267-288, 1996.

[13] S. Qin, Y. D. Zhang, and M. G. Amin, "Generalized coprime array configurations for direction-of-arrival estimation," in preparation. 